\title{
BMJ Open Effect of lateral positioning on the bronchial cuff pressure of a left-sided double-lumen endotracheal tube during thoracic surgery: study protocol for a prospective observational study
}

\author{
Eugene Kim, ${ }^{1}$ In-Young Kim, ${ }^{2}$ Sung-Hye Byun ${ }^{2}$
}

To cite: Kim E, Kim I-Y, Byun S-H. Effect of lateral positioning on the bronchial cuff pressure of a left-sided double-lumen endotracheal tube during thoracic surgery: study protocol for a prospective observational study. BMJ Open 2019;9:e026606. doi:10.1136/ bmjopen-2018-026606

- Prepublication history and additional material for this paper are available online. To view these files, please visit the journal online (http://dx.doi. org/10.1136/bmjopen-2018026606).

Received 11 September 2018 Revised 16 February 2019 Accepted 28 February 2019

Check for updates

(C) Author(s) (or their employer(s)) 2019. Re-use permitted under CC BY-NC. No commercial re-use. See rights and permissions. Published by BMJ.

${ }^{1}$ Department of Anesthesiology and Pain Medicine, Hanyang University College of Medicine, Seoul, Republic of Korea ${ }^{2}$ Department of Anesthesiology and Pain Medicine, Catholic University of Daegu School of Medicine, Daegu, Republic of Korea

Correspondence to Dr Sung-Hye Byun; stone0311@cu.ac.kr

\section{ABSTRACT}

Introduction Correct pressure is important when using a double-lumen endotracheal tube (DLT), especially in thoracic surgery. An inadequate bronchial cuff pressure (BCP) can cause air leak and interfere with visualisation of the surgical field, whereas an excessive pressure BCP can lead to cuff-related complications. Based on several reports that cuff pressure could alter after a positional change when using an endotracheal tube, we hypothesise that a change from the supine position to the lateral decubitus position, which is essential for thoracic surgery, would affect the BCP of the DLT.

Methods and analysis This prospective, single-centre, observational study will enrol 74 patients aged 18-70 years undergoing elective lung surgery from September 2018 to April 2019. The primary outcome will be the change in the 'initially established BCP' (maximum BCP not exceeding $40 \mathrm{~cm} \mathrm{H}_{2} \mathrm{O}$ with no air leak in the supine position) after lateral decubitus positioning. BCP and air leak will be assessed in each patient position during inflation of the cuff with air in $0.5 \mathrm{~mL}$ increments from 0 to $3 \mathrm{~mL}$. Secondary outcomes will include the incidence

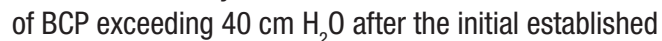
value and that of a change in the smallest bronchial cuff volume without air leak after a change to the lateral position. The relationship between the change in BCP and airway pressure, compliance and body mass index after lateral positioning will be investigated

Ethics and dissemination The study will be conducted in accordance with the Declaration of Helsinki and supervised by the Daegu Catholic University Medical Center institutional review board (study approval number CR-18-111). All patients will receive information about the study and will need to provide written informed consent before enrolment. The results will be presented at an international meeting and published in a peer-reviewed journal.

Trial registration number NCT03656406; Pre-results.

\section{INTRODUCTION}

It is important to maintain a correct cuff pressure when placing an endotracheal tube (ETT) in the trachea in patients undergoing
Strengths and limitations of this study

- Although there have been several studies of changes in the cuff pressure of a single-lumen endotracheal tube in response to a change in patient position, this will be the first to investigate whether the bronchial cuff pressure (BCP) of a double-lumen tube is affected by lateral positioning.

- Factors suspected to influence BCP will be identified. An understanding of these factors may aid the future management of BCP during changes in patient position.

- When equilibrating the cuff manometer and measuring BCP, manual deflation and inflation of the bronchial cuff with $0.5 \mathrm{~mL}$ of air may cause slight measurement errors.

- BCP and other study variables will be recorded until the start of surgery only, so the study will be unable to determine how BCP may be affected by other factors, for example, manipulation of the lung intraoperatively.

- The sample size was calculated for the primary outcome, that is, any change in BCP during lateral positioning, and may be too small for analysis of the secondary outcomes.

general anaesthesia. The usual recommendation is to maintain a pressure of $20-30 \mathrm{~cm} \mathrm{H}_{2} \mathrm{O}$ to prevent cuff-related complications, such as micro-aspiration or airway trauma. ${ }^{1-5}$ Correct pressure is also important when using doublelumen endotracheal tubes (DLTs) in thoracic surgery. ${ }^{6}$ Excessive pressure damages the mucosa of the tracheobronchial tree ${ }^{6}$ and various values in the range of $30-58 \mathrm{~cm} \mathrm{H}_{2} \mathrm{O}$ have been recommended as the upper limit for cuff pressure. ${ }^{257}$ However, air leak caused by underinflation of the bronchial cuff of the DLT can interfere with surgery by obstructing the surgeon's view of the operative field, ${ }^{8}$ particularly in video-assisted thoracoscopic surgery (VATS). Therefore, the initial 
bronchial cuff pressure (BCP) that can be effectively sealed to avoid air leak around the cuff while avoiding bronchial damage has been set at $35 \mathrm{~cm} \mathrm{H}_{2} \mathrm{O} .{ }^{9-11}$

Caution is needed after the correct BCP is initially established. Several studies have demonstrated alterations in the cuff pressure of the ETT in response to repositioning of the patient. The cuff pressure of singlelumen ETTs has been found to change when patients undergoing general surgery are moved from the supine position to the prone ${ }^{12}$ or lateral frank ${ }^{13}$ position and when the head is shifted from the neutral position to another position. ${ }^{14}{ }^{15}$ Such changes in cuff pressure have been reported to occur primarily because of displacement of the tube within the trachea. ${ }^{13}$ However, in a study performed in patients undergoing laparoscopic surgery, gravity-related conformational changes in the trachea (resulting from a combination of $\mathrm{CO}_{2}$ insufflation and head-down tilt) were attributed to increased cuff pressure. ${ }^{1}$ Therefore, a change in cuff pressure could be brought about by not only displacement of the tube but also the change in morphology of the trachea in response to a change in body positioning.

At present, there is no published research on whether the BCP of the DLT is affected by a change in patient positioning. We hypothesise that a change from the supine position to the lateral decubitus position, which is essential for thoracic surgery, would affect the BCP of the DLT, even when the problem of tube displacement has been eliminated by fibre-optic confirmation that the bronchial cuff is correctly positioned. The purpose of this study is to evaluate the effect of a change in patient positioning on the BCP of the DLT by observing the pressure of the bronchial cuff before and after a shift to the lateral decubitus position in patients undergoing thoracic surgery.

\section{METHODS AND ANALYSIS \\ Study design}

This prospective, single-centre, observational study is being conducted at the tertiary university hospital in Daegu, South Korea, from September 2018 to April 2019. It will be reported according to the Strengthening the Reporting of Observational Studies in Epidemiology statement (online additional files 1).

\section{Study population}

Patients aged 18-70 years and with an American Society of Anesthesiologists physical status of 1 or 2 who are scheduled to undergo elective lung surgery requiring lateral decubitus positioning and one-lung ventilation (OLV) using a left-sided DLT will be enrolled. The following exclusion criteria will be applied: need for a right-sided DLT; an intraluminal lesion in the left main bronchus (LMB); an anatomical problem in the tracheobronchial tree; lung impairment, such as chronic obstructive pulmonary disease; and refusal to participate in the study. A researcher (I-YK) will contact patients who are potential candidates for the study at the time of their preoperative visit. After providing information regarding the purpose, procedures and potential risks and benefits of the study, each prospective study participant will be asked to sign informed consent.

\section{Baseline demographics and surgical details}

The baseline characteristics of the study population will be collected preoperatively. The data collected will include age, sex, height, weight, body mass index (BMI, $\mathrm{kg} / \mathrm{m}^{2}$ ), size of the LMB, operative side (right or left) and the angle at which the operating table will be tilted.

\section{Anaesthesia and DLT intubation}

Standard monitoring, including ECG, non-invasive blood pressure measurement and pulse oximetry, will be performed after arrival in the operating room in all cases. A disposable bispectral index sensor (BIS, Aspect Medical Systems, Newton, Massachusetts, USA) will be used to monitor the depth of anaesthesia. All patients will undergo induction of anaesthesia with propofol and remifentanil using target-controlled infusion based on bispectral index monitoring of depth of anaesthesia and will receive rocuronium $0.8 \mathrm{mg} / \mathrm{kg}$ as a muscle relaxant to facilitate tracheal intubation. A disposable left-sided polyvinyl chloride DLT (Broncho-Cath, Mallinckrodt Medical Ltd., Athlone, Ireland) will be used for OLV in all patients. The size of the DLT will be chosen according to the diameter of the patient's LMB as measured on a CT scan of the chest (35 French for $<11 \mathrm{~mm}, 37$ French for $11-12 \mathrm{~mm}$ and 39 French for $\geq 12 \mathrm{~mm}){ }^{8}$ All patients will be intubated with the DLT under direct laryngoscopy, and correct positioning of the DLT in the LMB will be checked by fibre-optic bronchoscopy (FOB; Olympus Optical Co., Tokyo, Japan). Accurate placement of the DLT will be confirmed under the FOB view using the method described by Campos. ${ }^{16}$ A blue-coloured bronchial cuff will be positioned just below the carina without herniation when inflating the DLT cuff. After confirmation that the DLT is positioned correctly, the tube will be fixed temporarily at the patient's mouth using tape.

\section{Measurement of outcomes and data collection Bronchial cuff pressure}

Given that the aim of this study is to investigate the effect of a change from the supine to lateral position on BCP, the pressure will be measured by a cuff manometer (VBM Medizintechnik GmbH, Sulz, Germany) at a series of time points in the supine and lateral decubitus positions. A cuff manometer will be connected to the valve of the pilot balloon of the bronchial cuff via a three-way stopcock. The pressure measurements will start $2 \mathrm{~min}$ after confirmation of DLT placement in each patient position. The pressure of the tracheal cuff will be modified using a cuff manometer to maintain a pressure in the range of $20-30 \mathrm{~cm} \mathrm{H}_{2} \mathrm{O}$, and the volume of air administered will be recorded. The BCP will then be assessed while inflating the cuff with air in $0.5 \mathrm{~mL}$ increments from 0 to $3 \mathrm{~mL}$. Before injection of 
air into the bronchial cuff, the intracuff pressure will be equilibrated at atmospheric pressure by keeping the three-way stopcock open to the outside in order to maintain the resting volume of the cuff. If the pressure has a range of values, we will calculate the average of the maximum and minimum values and record it as the BCP. If the BCP exceeds $40 \mathrm{~cm} \mathrm{H}_{2} \mathrm{O}$ during expansion in $0.5 \mathrm{~mL}$ increments from 0 to $3 \mathrm{~mL}$, inflation of the bronchial cuff with air will be stopped and the bronchial cuff volume (BCV) and BCP will be recorded up to the last numerical value. Reports of the value that is acceptable as the upper limit of BCP for the DLT vary from $30 \mathrm{~cm} \mathrm{H}_{2} \mathrm{O}$ to $44 \mathrm{~cm} \mathrm{H}_{2} \mathrm{O} .^{9-11}$ Therefore, we have set an upper limit of $40 \mathrm{~cm} \mathrm{H}_{2} \mathrm{O}$ for the BCP in this study. Air leak will be monitored in both patient positions, so pressures will be measured under OLV by clamping the tracheal or bronchial lumen (operative side) of the DLT even in the supine position. The ventilation mode and setting will be kept constant in both patient positions during OLV to observe the effect of positional change on peak inspiratory pressure (PIP) and mean inspiratory pressure (Pmean), which may affect the pressure of the cuff. Ventilation will be maintained at a tidal volume of $6-8 \mathrm{~mL} / \mathrm{kg}$ body weight and the respiration rate will be adjusted to maintain an end-tidal carbon dioxide $\left(\mathrm{EtCO}_{2}\right)$ value of $30-35 \mathrm{~mm} \mathrm{Hg}$ and a positive end-expiratory pressure (PEEP) of $5 \mathrm{~cm} \mathrm{H}_{2} \mathrm{O}$.

\section{Air leak and respiratory variables}

As mentioned earlier, whether or not there is an air leak around the bronchial cuff will be assessed at each time point while inflating the cuff in $0.5 \mathrm{~mL}$ increments. Various methods can be used to detect air leak, ${ }^{17}$ including bubbling air into water and the $\mathrm{EtCO}_{2}$ waveform. ${ }^{818-20}$ However, there has been a suggestion that the minimum BCV needed to achieve an air seal using these techniques could be different from (usually larger than) the minimum volume that triggers collapse of the lung in the surgical field, ${ }^{17}$ and this is consistent with our unpublished experience. Air leak can also be tested simply by capnography or the pressure-volume loop seen on the respiratory monitor on the anaesthetic machine ${ }^{910}$ or by measuring the exhaled return tidal volume (VTe). ${ }^{21} \mathrm{We}$ will use these simple methods, and if the configuration on capnography or the pressure-volume loop is distorted and VTe is delivered at less than $80 \%$ of the established TV (TVset), an air leak will be considered to be present. TVset, VTe, PIP, Pmean and PEEP will be recorded at each time point, and a check will be performed for air leak.

\section{Determination of maximum and minimum BCP and BCV}

- The maximum pressure at which the BCP does not exceed $40 \mathrm{~cm} \mathrm{H}_{2} \mathrm{O}$ without air leak and the volume at that point in time will be denoted as the "maximum BCP' and 'maximum BCV', respectively, and as the 'initial established BCP' and 'initial established BCV' if in the supine position
- The smallest BCV without air leak and the pressure at that time point will be denoted as 'minimum BCV' and 'minimum BCP'.

After measuring these values, the bronchial cuff will be aspirated completely and equilibrated at atmospheric pressure, thereby returning to its resting state under two-lung ventilation.

\section{Length of the LMB}

The length of the LMB will be measured before and after lateral positioning to check for a conformational change. As described in our previous report, ${ }^{22}$ when the tip of the FOB reaches the tracheal or bronchial carina, the point of contact with the elbow connector of the DLT will be marked on the tape previously attached to the shaft of the FOB, and the length between these two markers will be taken as the length of the LMB. All the FOB procedures, including confirmation of the correct position of the DLT and measurement of length, will be performed by two experienced investigators (EK, S-HB) with at least 5 years of experience in thoracic anaesthesia.

\section{Repeated outcomes measurements after lateral positioning}

Outcomes measurements, as mentioned above, will be repeated in the lateral decubitus position and at the start of the operation. The patient will be placed in the lateral decubitus position with an axillary roll under the dependent axilla, and the operating table will be flexed enough to allow the shoulder and hip to be placed on the horizontal line and the intercostal space to be widened maximally. All the positioning procedures will be undertaken by an independent senior resident. Whether the position of the DLT, that is, the bronchial cuff position just below the carina without herniation, is the same as that before moving the patient into the lateral position will be determined by the same investigator and the length of the LMB will be measured using another FOB. The tracheal cuff will be inflated using the administered volume of air in the supine position. The cuff pressure will then be checked using a cuff manometer and modified to maintain pressure in the range of $20-30 \mathrm{~cm} \mathrm{H}_{2} \mathrm{O}$ if necessary. Like in the supine position, the BCP and air leak will then be checked while expanding the cuff from 0 to $3 \mathrm{~mL}$ in $0.5 \mathrm{~mL}$ increments as long as the $\mathrm{BCP}$ does not exceed $40 \mathrm{~cm} \mathrm{H}_{2} \mathrm{O}$. The maximum and minimum BCP and BCV and the TVset, VTe, PIP, Pmeanand PEEP will be recorded in the lateral decubitus position at each time point. After the maximum $\mathrm{BCP}$ is recorded, the $\mathrm{BCP}$ will be lowered to the minimum value; the cuff manometer will remain connected to the pilot balloon until the start of the operation. Two minutes after incision and insertion of the trocar for thoracoscopy, the BCP measured by the cuff manometer will be recorded and whether the operative lung collapses correctly in the operative field will be recorded by the operating surgeon. Next, the higher BCP values will be measured and recorded in $0.5 \mathrm{~mL}$ increments up to the maximum BCP, which will be maintained throughout the operation. 


\section{Primary and secondary outcomes}

The primary outcome of this study will be the change in 'initial established BCP' (maximum BCP established initially in the supine position that does not exceed 40 $\mathrm{cm} \mathrm{H}_{2} \mathrm{O}$ with no air leak) after lateral decubitus positioning, that is, the difference in BCP between the supine and lateral positions when injecting the initial established BCV' (maximum BCV established in the supine position).

The secondary outcomes will include the proportion of

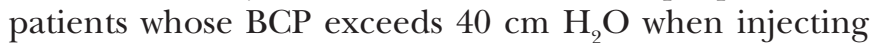
the 'initial established BCV' and the proportion with a change (decrease or increase) in the minimum BCV without air leak after lateral positioning. An increase in the minimum BCV would mean that the minimum BCV in the supine position would cause an air leak in the lateral position. Analysing this relationship may help in the management of BCP in the future. The relationship between the change in BCP and other clinical variables, including airway pressure (PIP, Pmean), airway compliance $\left(\mathrm{C}_{\mathrm{dyn}}=\frac{\mathrm{TVe}}{\mathrm{PIP}-\mathrm{PEEP}}\right)$, and BMI after lateral positioning, will be analysed.

\section{Sample size}

The primary outcome of the study will be the change in 'initial established BCP' in the supine and lateral positions. In a preliminary study, the difference in BCP when injecting the 'initial established BCV' between the two positions was 2.3 and the SD was 6.53 . Based on that result, we calculated that 66 patients would be required to achieve a power of $80 \%$ and a significance level of $5 \%$ (two-sided). Seventy-four patients will be included to allow for a $10 \%$ dropout rate.

\section{Statistical analysis}

The data will be analysed on an intention-to-treat basis, and missing data will be handled using the last observation carried forward method. The distribution of the data will be checked for normality using the Kolmogorov-Smirnov test. Normally distributed data will be presented as the mean \pm SD deviation and analysed using the paired t-test. Data that are not distributed normally will be expressed as the median (IQR) and analysed using the Wilcoxon signed-rank test. Categorical data will be expressed as the number (percentage). Pearson's correlations will be calculated to determine the relationships between airway pressure, compliance, BMI and the change in BCP after lateral positioning. A p value $<0.05$ will be considered statistically significant. All statistical analyses will be performed using IBM SPSS Statistics V.19.0.0 (IBM Corp.).

\section{Patient and public involvement}

No patients or members of the public were involved in the design of the study. Patients in this study can request the results from the research team if they are interested.

\section{Ethics and dissemination}

The study will be conducted in accordance with the principles of the Declaration of Helsinki. The institutional review board and all study participants will be notified if any important protocol modifications or violations arise. The results of the study will be presented at an international congress and published in a peer-reviewed journal. The data collected will also be made available in a public data repository.

\section{DISCUSSION}

This study has been designed as an observational study where the investigators will perform thoracic anaesthesia as per routine practice, without the intended intervention, and only observe the outcomes. Although measurement of the cuff pressure is not a routine practice, the overall procedure, including confirmation of the DLT position and repositioning of the DLT under the FOB after lateral positioning of the patients, follows the standard protocol for thoracic anaesthesia. With regard to measurement of the ETT cuff pressure, it is already known that connection of the manometer to the pilot balloon results in loss of cuff pressure. ${ }^{23}$ Unfortunately, DLT displacement is very common after a change in the patient's posture, and deflation or inflation of the tracheal and bronchial cuffs using the syringe should be repeated each time the DLT position changes. Therefore, we will inject the same volume of air into the cuff in order to create equal conditions in both patients' posture and decided to measure the BCP by addition of air in $0.5 \mathrm{~mL}$ increments from 0 to $3 \mathrm{~mL}$.

\section{Limitations}

This protocol has some limitations. First, manipulation of the cuff manometer or manual deflation and inflation of the cuff with the established volume of air for equilibrating the intracuff pressure with the atmospheric pressure or measuring the tracheal and BCPs will inevitably result in slight measurement errors. Second, the cuff pressure and other study variables will be checked only until the start of surgery, because there have been wide variations in the operating time during VATS at our institution. Such variations can complicate accurate evaluation of the postoperative outcomes. Furthermore, prolonged surgery involving considerable surgical manipulation of the lungs or bronchi can cause airway oedema or mucus hypersecretion and affect the cuff pressure or other respiratory variables. If this limitation can be overcome in the future, we will perform further studies of the postoperative outcomes to determine the effects of other factors on the BCP. Third, the same investigator will be observing the DLT position at two time points (the supine position and the lateral position) in order to confirm whether the DLT is placed at the same site within the LMB. In order to reduce the risk of bias, at least two investigators will be involved, and measurement of the LMB length will be repeated using another FOB. Finally, the sample size was calculated for the primary outcome, that is, the change in the BCP during lateral positioning. Accordingly, it may be insufficient for analysis of the secondary outcomes. 
Contributors EK and S-HB drafted the manuscript. EK, I-YK and S-HB will be responsible for the screening and enrolment of patients. S-HB revised this manuscript and will supervise the study. All authors have read and approved the final version of this manuscript.

Funding This work will be supported by a grant from the Research Institute of Medical Science, Catholic University of Daegu (grant number 201809). The funder has no role in the study, including in the study design or the decision to submit this manuscript for publication. It will also have no involvement in management, analysis or interpretation of the data collected in the study.

Competing interests None declared.

Patient consent for publication Not required.

Ethics approval The study protocol has been approved by the Daegu Catholic University Medical Center institutional review board (CR-18-111).

Provenance and peer review Not commissioned; externally peer reviewed.

Open access This is an open access article distributed in accordance with the Creative Commons Attribution Non Commercial (CC BY-NC 4.0) license, which permits others to distribute, remix, adapt, build upon this work non-commercially, and license their derivative works on different terms, provided the original work is properly cited, appropriate credit is given, any changes made indicated, and the use is non-commercial. See: http://creativecommons.org/licenses/by-nc/4.0/.

\section{REFERENCES}

1. Wu CY, Yeh YC, Wang MC, et al. Changes in endotracheal tube cuff pressure during laparoscopic surgery in head-up or head-down position. BMC Anesthesiol 2014;14:75.

2. Seegobin RD, van Hasselt GL. Endotracheal cuff pressure and tracheal mucosal blood flow: endoscopic study of effects of four large volume cuffs. Br Med J 1984;288:965-8.

3. Sengupta P, Sessler DI, Maglinger P, et al. Endotracheal tube cuff pressure in three hospitals, and the volume required to produce an appropriate cuff pressure. BMC Anesthesiol 2004;4:8.

4. Athiraman U, Gupta R, Singh G. Endotracheal cuff pressure changes with change in position in neurosurgical patients. Int J Crit IIIn Inj Sci 2015;5:237-41.

5. Joh S, Matsuura $\mathrm{H}$, Kotani $\mathrm{Y}$, et al. Change in tracheal blood flow during endotracheal intubation. Acta Anaesthesiol Scand 1987;31:300-4.

6. Brodsky JB. Lung separation and the difficult airway. $\mathrm{Br} J$ Anaesth 2009;103(Suppl 1):i66-75.

7. Lewis FR, Schiobohm RM, Thomas AN. Prevention of complications from prolonged tracheal intubation. Am J Surg 1978;135:452-7.

8. Hannallah M, Benumof JL, Silverman PM, et al. Evaluation of an approach to choosing a left double-lumen tube size based on chest computed tomographic scan measurement of left mainstem bronchial diameter. J Cardiothorac Vasc Anesth 1997;11:168-71.

9. Araki K, Nomura R, Urushibara R, et al. Displacement of the double-lumen endobronchial tube can be detected by bronchial cuff pressure change. Anesth Analg 1997;84:1349-53.

10. Araki K, Nomura R, Urushibara R, et al. Bronchial cuff pressure change caused by left-sided double-lumen endobronchial tube displacement. Can J Anaesth 2000;47:775-9.

11. Slinger PD, Chripko D. A clinical comparison of bronchial cuff pressures in three different designs of left double-lumen tubes. Anesth Analg 1993;77:305-8.

12. Kim D, Jeon B, Son JS, et al. The changes of endotracheal tube cuff pressure by the position changes from supine to prone and the flexion and extension of head. Korean J Anesthesiol 2015;68:27-31.

13. Kim HC, Lee YH, Kim E, et al. Comparison of the endotracheal tube cuff pressure between a tapered- versus a cylindrical-shaped cuff after changing from the supine to the lateral flank position. Can $\mathrm{J}$ Anaesth 2015;62:1063-70.

14. Lizy C, Swinnen W, Labeau S, et al. Cuff pressure of endotracheal tubes after changes in body position in critically ill patients treated with mechanical ventilation. Am J Crit Care 2014;23:e1-8.

15. Choi E, Park Y, Jeon Y. Comparison of the cuff pressure of a TaperGuard endotracheal tube and a cylindrical endotracheal tube after lateral rotation of head during middle ear surgery: a single-blind, randomized clinical consort study. Medicine 2017;96:e6257.

16. Campos JH. Update on tracheobronchial anatomy and flexible fiberoptic bronchoscopy in thoracic anesthesia. Curr Opin Anaesthesiol 2009;22:4-10.

17. Hannallah MS, Benumof JL, McCarthy PO, et al. Comparison of three techniques to inflate the bronchial cuff of left polyvinylchloride double-lumen tubes. Anesth Analg 1993;77:990-4.

18. Seo JH, Bae J, Paik H, et al. Computed tomographic window setting for bronchial measurement to guide double-lumen tube size. $J$ Cardiothorac Vasc Anesth 2018;32:863-8.

19. Fisicaro MD, Maguire DP, Armstead VE. Using the capnograph to confirm lung isolation when using a bronchial blocker. $J$ Clin Anesth 2010;22:557-9.

20. Brodsky JB, Lemmens HJ. Left double-lumen tubes: clinical experience with 1,170 patients. J Cardiothorac Vasc Anesth 2003;17:289-98.

21. Rozycki SK, Dixon FP, Yopp MA, et al. Endotracheal Tube Seal and Suction Performance in a Novel Biorealistic Tracheal Model. Respir Care 2015;60:1113-9.

22. Byun $\mathrm{SH}$, Kang SH, Kim JH, et al. Comparison of a tube-holder (Rescuefix) versus tape-tying for minimizing double-lumen tube displacement during lateral positioning in thoracic surgery: $A$ prospective, randomized controlled study. Medicine 2016;95:e4486.

23. Asai S, Motoyama A, Matsumoto $Y$, et al. Decrease in cuff pressure during the measurement procedure: an experimental study. $J$ Intensive Care 2014;2:34. 